\title{
(2) OPEN ACCESS \\ Longitudinal changes of spinal cord grey and white matter following spinal cord injury
}

\author{
Gergely David (D) ,' Dario Pfyffer (D) , ${ }^{1}$ Kevin Vallotton, ${ }^{1}$ Nikolai Pfender, ${ }^{1}$ \\ Alan Thompson, ${ }^{2}$ Nikolaus Weiskopf, ${ }^{3,4}$ Siawoosh Mohammadi, ${ }^{5}$ Armin Curt, ${ }^{1}$ \\ Patrick Freund (1) 1,2,3,6
}

\begin{abstract}
Spinal Cord Injury Center, Balgrist University Hospital, University of Zurich, Zurich, Switzerland

${ }^{2}$ Department of Brain Repair and Rehabilitation, UCL Institute of Neurology, London, UK ${ }^{3}$ Department of Neurophysics, Max Planck Institute for Human Cognitive and Brain Sciences, Leipzig, Germany

${ }^{4}$ Felix Bloch Institute for Solid State Physics, Faculty of Physics and Earth Sciences, Leipzig University, Leipzig, Germany ${ }^{5}$ Department of Systems Neuroscience, University Medical Center HamburgEppendorf, Hamburg, Germany ${ }^{6}$ Wellcome Trust Centre for Neuroimaging, UCL Institute of Neurology, London, UK
\end{abstract}

Correspondence to

Professor Patrick Freund, Spinal Cord Injury Center, Balgrist University Hospital, Zurich 8008 Switzerland; patrick.freund@ balgrist.ch

Received 9 February 2021 Accepted 9 June 2021 Published Online First 31 July 2021

\section{Check for updates}

(c) Author(s) (or their employer(s)) 2021. Re-use permitted under CC BY. Published by BMJ.

To cite: David G, Pfyffer D, Vallotton K et al. J Neurol Neurosurg Psychiatry 2021:92:1222-1230.
ABSTRACT
Objectives Traumatic and non-traumatic spinal cord injury produce neurodegeneration across the entire neuraxis. However, the spatiotemporal dynamics of spinal cord grey and white matter neurodegeneration above and below the injury is understudied.

Methods We acquired longitudinal data from 13 traumatic and 3 non-traumatic spinal cord injury patients (8-8 cervical and thoracic cord injuries) within 1.5 years after injury and 10 healthy controls over the same period. The protocol encompassed structural and diffusionweighted MRI rostral (C2/C3) and caudal (lumbar enlargement) to the injury level to track tissue-specific neurodegeneration. Regression models assessed group differences in the temporal evolution of tissue-specific changes and associations with clinical outcomes.

Results At 2 months post-injury, white matter area was decreased by $8.5 \%$ and grey matter by $15.9 \%$ in the lumbar enlargement, while at C2/C3 only white matter was decreased (-9.7\%). Patients had decreased cervical fractional anisotropy (FA: $-11.3 \%$ ) and increased radial diffusivity $(+20.5 \%)$ in the dorsal column, while FA was lower in the lateral $(-10.3 \%)$ and ventral columns $(-9.7 \%)$ of the lumbar enlargement. White matter decreased by $0.34 \%$ and $0.35 \%$ per month at C2/C3 and lumbar enlargement, respectively, and grey matter decreased at C2/C3 by $0.70 \%$ per month.

Conclusions This study describes the spatiotemporal dynamics of tissue-specific spinal cord neurodegeneration above and below a spinal cord injury. While above the injury, grey matter atrophy lagged initially behind white matter neurodegeneration, in the lumbar enlargement these processes progressed in parallel. Tracking trajectories of tissue-specific neurodegeneration provides valuable assessment tools for monitoring recovery and treatment effects.

\section{INTRODUCTION}

Spinal cord injury (SCI) is a devastating event that usually leads to permanent impairments. ${ }^{1}$ Currently, there is no cure for SCI, but rehabilitation has been shown to improve outcome. ${ }^{2}$ Traumatic and nontraumatic SCI cause not only focal damage to the injury site (primary injury), ${ }^{3-5}$ but also trigger a cascade of secondary pathological processes that propagate above and below the injury site, ${ }^{167}$ affecting also the brain. ${ }^{8-11}$ However, little is known about the spatiotemporal dynamics of remote spinal cord white and grey matter atrophy, which are key processes involved in the patients' long-term recovery and targets of interventions. ${ }^{12} 13$

Experimental studies revealed early anterograde and retrograde neurodegeneration of the sensory and motor tracts above and below the injury. ${ }^{14} 15$ In patients with chronic traumatic SCI, significant tissue-specific atrophy and altered microstructure was detected both in the cervical ${ }^{16}$ and lumbar spinal cord. ${ }^{17}$ Starting at the acute stage, these remote macrostructural (atrophy) and microstructural changes have been shown to continuously progress over the first year after injury at $\mathrm{C} 2 /$ C3 level, ${ }^{8}{ }^{10}$ with cord atrophy decelerating after 2 years. ${ }^{18}$ In addition, there is evidence that the magnitude of remote cord atrophy and reduction in myelin (demyelination) is associated with clinical impairment. ${ }^{810}$ However, these studies focused only on remote degeneration rostral to the injury site by means of cross-sectional cord area, with no information on longitudinal tissue-specific changes. Based on the literature, we hypothesised that (i) grey and white matter microstructural and macrostructural changes are already present early after injury and (ii) continue to worsen over time above and below the level of injury.

\section{METHODS}

\section{Study participants}

Thirteen patients with traumatic and three with non-traumatic SCI (three females, age (mean \pm SD): $50.3 \pm 16.0$ years), admitted consecutively into the rehabilitation programme at the Balgrist University Hospital (Zurich, Switzerland) between January 2016 and March 2019, and ten healthy controls recruited during the same time period (three females, age: $45.3 \pm 19.2$ years) participated in this 1.5 -year longitudinal study (table 1 ). Inclusion criteria were (1) no pre-existing neurologic or mental disorders, (2) no history of head and brain lesions, (3) no MRI contraindications and (4) no pregnancy. Participants were scanned at baseline $(\mathrm{n}=14)$ and at $6(\mathrm{n}=12)$ and 16 months $(n=12)$ follow-up. In patients, the mean $( \pm S D)$ interval to the first scan following injury was 2.1 $( \pm 1.3)$ months, to the second scan $8.2( \pm 4.2)$ months and to the third scan 18.1 ( \pm 7.1$)$ months. The baseline scans of the healthy controls served as the healthy cohort in a previous cross-sectional study. $^{17}$ 


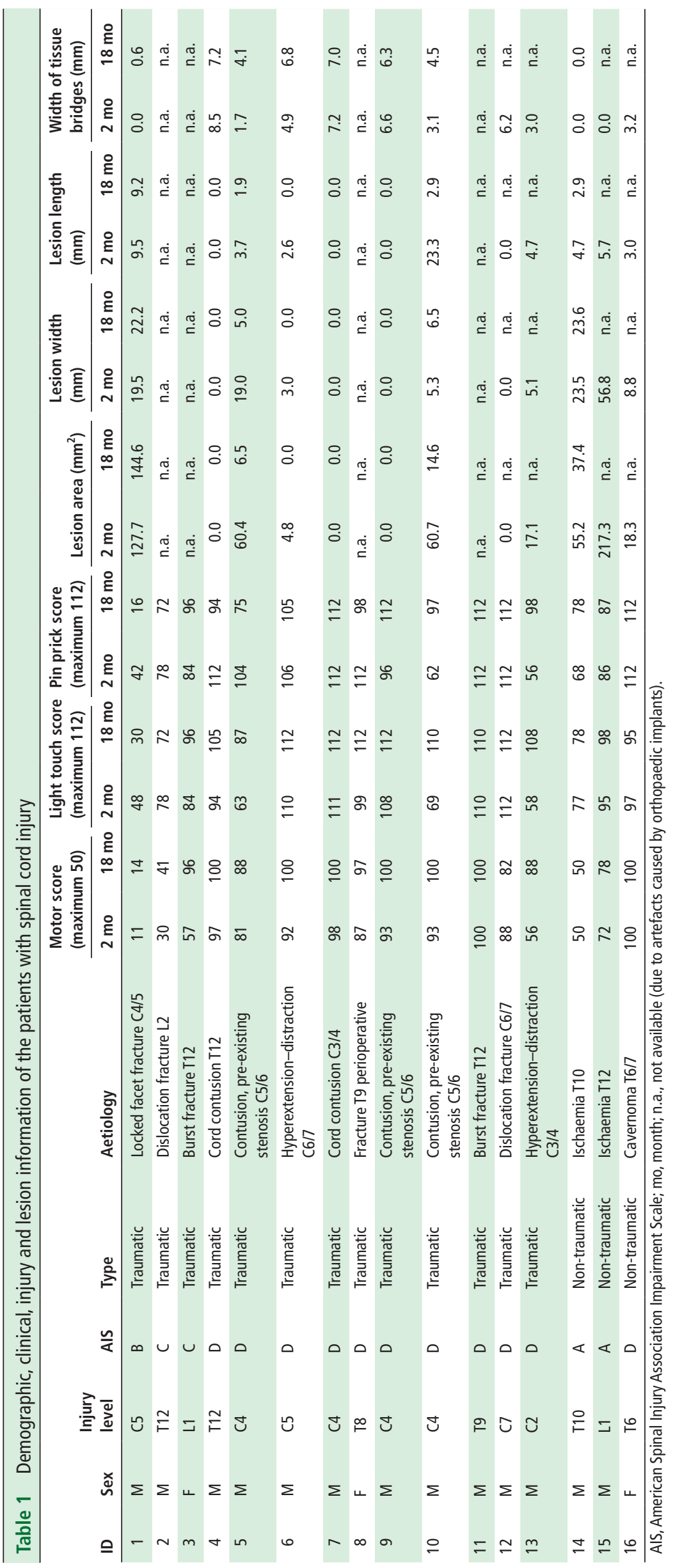



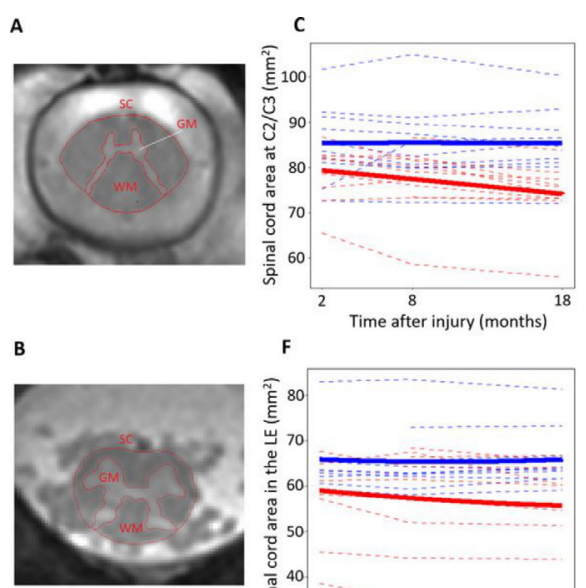

$\mathrm{F}$

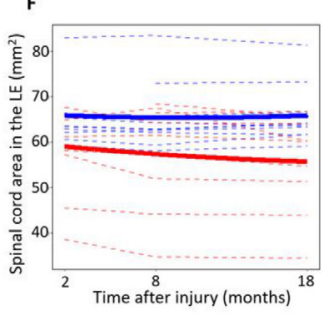

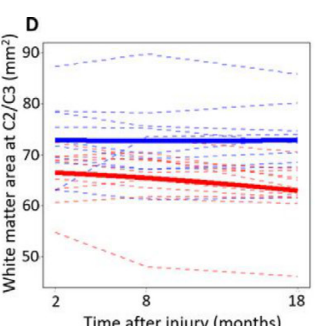

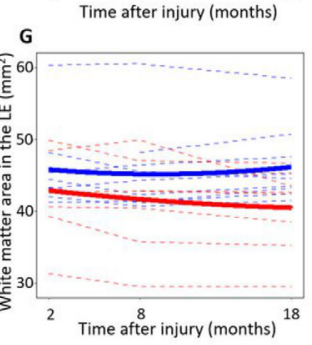

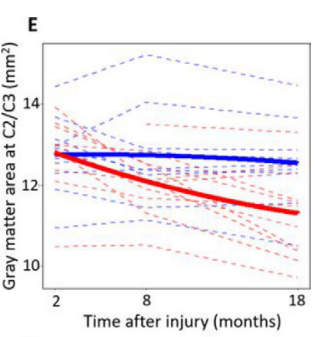

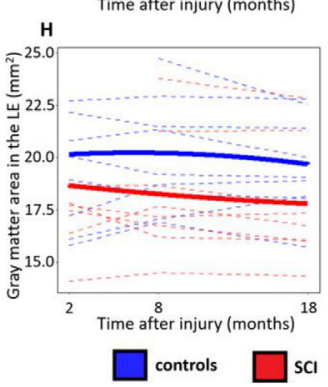

Figure 1 Longitudinal changes in cross-sectional areas. (A,B) Cross-sectional areas of spinal cord (SC), white matter (WM) and grey matter (GM) were measured at two locations, (A) above the injury at $C 2 / C 3$ and (B) below the injury in the lumbar enlargement (LE), by means of semiautomatic and manual segmentation. (C-H) Longitudinal changes in cross-sectional areas of SC, WM and GM in controls (blue lines) and patients with spinal cord injury (SCI) (red lines), over three timepoints within the first year after injury. Dashed lines represent evolution in individual subjects, while the solid lines represent the quadratic fit in controls and patients with $\mathrm{SCl}$ separately.

\section{MRI acquisition protocol}

All MRI measurements were done on a clinical 3T Siemens Skyra $^{\text {Fit }}$ system (Erlangen, Germany), using the standard transmit coil for transmission and the 16-channel head and neck coil and standard spine matrix coil for reception. Two anatomical locations were measured: the upper cervical cord and the lumbar enlargement located between T11-L1 (figure 1A,B). To identify the location of the lumbar enlargement, a sagittal T2-weighted two-dimensional (2D) turbo spin echo sequence of the lumbar region was acquired with the following parameters: 23 slices, slice thickness $=3 \mathrm{~mm}$, in-plane resolution $=0.6 \times 0.6 \mathrm{~mm}^{2}$, field of view $(\mathrm{FOV})=160 \times 160 \mathrm{~mm}^{2}$, echo time $(\mathrm{TE})=96 \mathrm{~ms}$, repetition time $(\mathrm{TR})=5510 \mathrm{~ms}$, flip angle $=150^{\circ}$, readout bandwidth $=283 \mathrm{~Hz} /$ pixel and acquisition time $=01: 57 \mathrm{~min}$.

At both anatomical locations, axial T2*-weighted structural images were acquired using a three-dimensional (3D) multiecho spoiled gradient echo sequence (Siemens Multi-Echo Data Image Combination (MEDIC)). In the upper cervical cord, the 20 axial-oblique slices were centred at the C2/C3 intervertebral disc, while in the lumbar cord they were centred at the widest point of the lumbar cord (lumbar enlargement) as appearing in the T2-weighted image. ${ }^{19}$ Four repetitions were acquired with the following parameters: slice thickness $=2.5 \mathrm{~mm}$, in-plane resolution $=0.5 \times 0.5 \mathrm{~mm}^{2}, \quad \mathrm{FOV}=192 \times 162 \mathrm{~mm}^{2}, \quad 5$ echoes, $\mathrm{TE}$ of first echo $=7 \mathrm{~ms}$, echo spacing $=3 \mathrm{~ms}, \mathrm{TR}=44 \mathrm{~ms}$, flip angle $=11^{\circ}$, readout bandwidth $=260 \mathrm{~Hz} /$ pixel, total acquisition time $=7: 16 \mathrm{~min}$.

Diffusion-weighted images for diffusion tensor imaging (DTI) were acquired using a reduced FOV single-shot spin echo Echo Planar Imaging (EPI) sequence with identical slice prescription as the T2*-weighed images and consisting of 60 diffusionweighted $\left(b=500 \mathrm{~s} / \mathrm{mm}^{2}\right)$ and 7 T2-weighted $\left(b=0 \mathrm{~s} / \mathrm{mm}^{2}\right)$ images. Acquisition parameters were: slice thickness $=5 \mathrm{~mm}$, in-plane resolution $=0.76 \times 0.76 \mathrm{~mm}^{2}, \quad F O V=133 \times 30 \mathrm{~mm}^{2}$, $\mathrm{TE}=73 \mathrm{~ms}, \mathrm{TR}=350 \mathrm{~ms}$, readout bandwidth $=768 \mathrm{~Hz} /$ pixel, $5 / 8$ partial Fourier in the phase-encoding direction. The acquisition was cardiac gated ( 3 slices per cycle, trigger delay $=200 \mathrm{~ms}$ ) and lasted approximately $8 \mathrm{~min}$, depending on the heart rate.
In patients, an additional sagittal T2-weighted 2D turbo spin echo sequence of the lesion area was acquired with the following parameters: 20 slices, slice thickness $=2.5 \mathrm{~mm}$, in-plane resolution $=0.3 \times 0.3 \mathrm{~mm}^{2}, \quad F O V=220 \times 200 \mathrm{~mm}^{2}, \quad T E=84 \mathrm{~ms}$, $\mathrm{TR}=3500 \mathrm{~ms}$, flip angle $=160^{\circ}$, readout bandwidth $=260 \mathrm{~Hz}$ / pixel, acquisition time $=1: 47 \mathrm{~min}$.

\section{Processing of $\mathrm{T} 2{ }^{*}$-weighted images}

An average of the four T2*-weighted structural images was created using serial longitudinal registration to account for within-scan motion, ${ }^{20}$ which was resliced to $5 \mathrm{~mm}$ slice thickness to increase signal-to-noise ratio. The resulting image was segmented for spinal cord using the semiautomatic $3 \mathrm{D}$ active surface method in JIM V7.0 software. ${ }^{21}$ Grey matter was segmented manually using subvoxel manual segmentation in JIM V7.0. White matter segmentation was obtained by subtracting grey matter from the spinal cord mask. Cross-sectional tissue areas including spinal cord area (SCA), grey matter area (GMA), and white matter area (WMA) were extracted from these segmentations and averaged across slices. In the lumbar enlargement, only three slices around the slice with the largest SCA were considered to ensure comparable and reproducible anatomical location.

\section{Processing of diffusion-weighted images}

Diffusion-weighted images underwent artefact correction using the SPM-based ACID toolbox. ${ }^{22}$ All images were cropped to an in-plane FOV of $30 \times 30 \mathrm{~mm}^{2}$ to exclude much of the non-spinal cord tissue. Eddy current and motion correction were applied using the Eddy Current and Motion Correction (ECMOCO) algorithm. ${ }^{23}$ The mean diffusion-weighted image was segmented for spinal cord using Propseg. ${ }^{24}$ DTI maps including fractional anisotropy (FA), axial diffusivity (AD), and radial diffusivity (RD) were obtained using the ACID robust-fitting algorithm. ${ }^{22} 25$ $\mathrm{AD}$ and $\mathrm{RD}$ represent the diffusivities parallel and perpendicular to the axons, respectively, under the assumption that the diffusion ellipsoid is aligned with the white matter tracts, which is usually the case in the spinal cord. FA is a measure of diffusion 
anisotropy, with a value of 0 representing isotropic and 1 representing full anisotropic diffusion. The mean diffusion-weighted image was spatially normalised to the PAM50 template using Spinal Cord Toolbox (V4.2.1), which shares the same coordinate system with the MNI-ICBM 152 template. ${ }^{26}$ The obtained deformation field was applied to all DTI maps. Mean values of $\mathrm{FA}, \mathrm{AD}$ and $\mathrm{RD}$ were extracted within three white matter tracts of interest (dorsal, lateral and ventral columns) using the atlas-based analysis capabilities of Spinal Cord Toolbox. ${ }^{27}$ Partial volume effects were minimised by extracting the 'maximum a posteriori' value instead of the average within the tracts, where voxels at the edges are underrepresented.

\section{Clinical assessments}

In patients, the neurological status and functional impairment were assessed according to the International Standards for Neurological Classification of Spinal Cord Injury (ISNCSCI) protocol. $^{28}$ ISNCSCI assesses the strength of five key muscles in the upper and lower extremities on both sides on a scale of 0-5 (0: no motor function, 5: normal function) and the sensory function of 28 dermatome pairs in terms of light touch sensation and sharp-dull discrimination (pinprick) on a scale of $0-2$ (0: no sensation/discrimination, 2 : normal sensation/discrimination). Single scores summed up across all neurological levels are referred to as total motor, light touch and pinprick scores. In addition, Spinal Cord Independence Measure (SCIM) was used to assess daily life independence on a scale between 0 and $100 .^{29}$

\section{Electrophysiological measurements}

The electrophysiological examinations, including motor evoked potentials (MEP) for tibialis anterior and somatosensory evoked potentials (SEP) of the posterior tibialis nerve, were conducted according to the standard protocol of the European multicenter study about SCI (https://www.emsci.org/).

\section{Lesion segmentation}

In patients, the lesion (hyperintense signal in the parenchyma) was segmented in the sagittal T2-weighted image. On the midsagittal slice, the following parameters were quantified as previously described ${ }^{4}$ : mid-sagittal anterior-posterior lesion width (defined as the maximal anterior-posterior width of the lesion), mid-sagittal rostrocaudal lesion length (defined as the maximal rostrocaudal length of the lesion), mid-sagittal lesion area and mid-sagittal width of tissue bridges.

\section{Statistical analysis}

Age and sex differences between patients and controls were assessed using Mann-Whitney $U$ test and Fisher exact test, respectively. In the lumbar enlargement, we excluded six patients with SCI and one healthy control due to motion artefacts and/ or signal dropout caused by orthopaedic implants. For assessing baseline macrostructural and microstructural differences, baseline SCA, GMA, WMA and DTI metrics within white matter tracts were compared using two-sample t-test (unpaired, one tailed, $\mathrm{p}<0.05$ ). In addition, voxel-based analysis was performed in SPM 12, using a two-sample t-test in each voxel, where the statistical parametric maps were thresholded at $\mathrm{p}<0.005$ (uncorrected), followed by a peak-level threshold of $p<0.05$ (familywise error (FWE) corrected).

For longitudinal changes in SCA, GMA, WMA and DTI metrics, a linear mixed effect model was implemented using the nlme package in $\mathrm{R}(\mathrm{p}<0.05)$. To investigate voxel-based longitudinal changes in the DTI maps, a similar linear mixed effect model with subject-varying intercept and rate of change (and allowing for interaction between groups) was implemented using the SwE toolbox. ${ }^{30}$ Voxels with $\mathrm{p}<0.05$ (false discovery rate (FDR) corrected) at peak level were considered significant.

To compare relative atrophies of grey versus white matter, standardisation was applied on the patients' GMA and WMA values. During standardisation, the average GMA (WMA) across controls was subtracted from the individual GMA (WMA), and the difference was divided by the SD of GMA (WMA) across controls. The resulting standardised score represents the number of SD by which the individual GMA (WMA) lies above or below the corresponding average value across all controls. A linear mixed effect model over time was applied on the individual differences between standardised GMA and WMA, where the intercept indicates baseline differences in the relative white and grey matter atrophy, and the slope indicates a linear change in relative atrophies over time.

We also investigated the ability of baseline ( 2 months post injury) MRI readouts to predict 1.5 -year clinical scores, independently of the baseline clinical values. For this, a multiple regression model was applied in each voxel with the baseline clinical scores and voxel-wise baseline MRI readouts being the two predictors and the 1.5-year clinical score being the dependent variable. The initial cluster-defining threshold of $p=0.005$ was followed by a peak-level threshold of $\mathrm{p}<0.05$ (FWE corrected).

\section{RESULTS}

\section{Patients' characteristics and clinical outcomes}

Of the 16 patients studied, 8 were tetraplegic and 8 paraplegic. Two patients were American Spinal Injury Association Impairment Scale (AIS) A ('complete'), 1 AIS B ('sensory incomplete'), 2 AIS C ('motor incomplete'), and 11 AIS D ('motor incomplete') (table 1). For ISCNSCI and SCMI scores, a logarithmic fit with respect to time on the longitudinal values was superior to a linear fit, as shown by similar or lower $\mathrm{p}$ values of the slope, implying that recovery rate is faster soon after injury and decreases over time. Over 1.5 years post injury, patients recovered by 1.5 points per log month $(95 \%$ CI: 0.6 to 2.3 ) on their ISNCSCI upper extremity motor score $(p=0.002)$, and showed weak tendencies to improvement by 2.4 points $(-0.8$ to $5.6, \mathrm{p}=0.131)$ on their lower extremity motor score and by 3.6 points $(-0.8$ to $8.0, \mathrm{p}=0.101)$ on the light touch score. Pinprick scores did not change significantly over time $(p=0.718)$. In the same period, SCIM score improved by 10.5 points per log month (3.5 to $17.5, \mathrm{p}=0.005)$. There was no difference between patients and controls in terms of age $(\mathrm{W}=66, \mathrm{p}=0.476)$. Electrophysiological characterisation demonstrated myelopathy in 13 of 16 patients, showing pathological tibial nerve SEP ( 3 normal, 6 delayed, 7 abolished) and/or pathological MEP recorded from tibialis anterior (motoneuron pool L4/5) and abductor hallucis muscle (motoneuron pool S1/S2) resulting in pathologic central motor conduction time (4 normal, 6 delayed, 6 abolished MEP). All motor complete paraplegic patients (AIS A-B) showed completely abolished SEP and MEP of the tibialis anterior muscles, whereas three patients with incomplete tetraplegia or paraplegia (AIS C-D) exhibited normal values.

\section{Lesion characteristics}

Mid-sagittal tissue bridges were present in all motor incomplete patients (AIS C-D), with a mean $( \pm S D)$ width of $4.9 \pm 2.3 \mathrm{~mm}$ at 2 months post injury, which showed no significant change over time $(\mathrm{p}=0.312)$. Of the 13 motor incomplete patients, 4 had no visible lesions, while the lesion could not be assessed in 4 patients due to metal artefacts. Motor complete patients (AIS A-B) did not have preserved mid-sagittal tissue bridges. In patients with visible lesions, the mean 


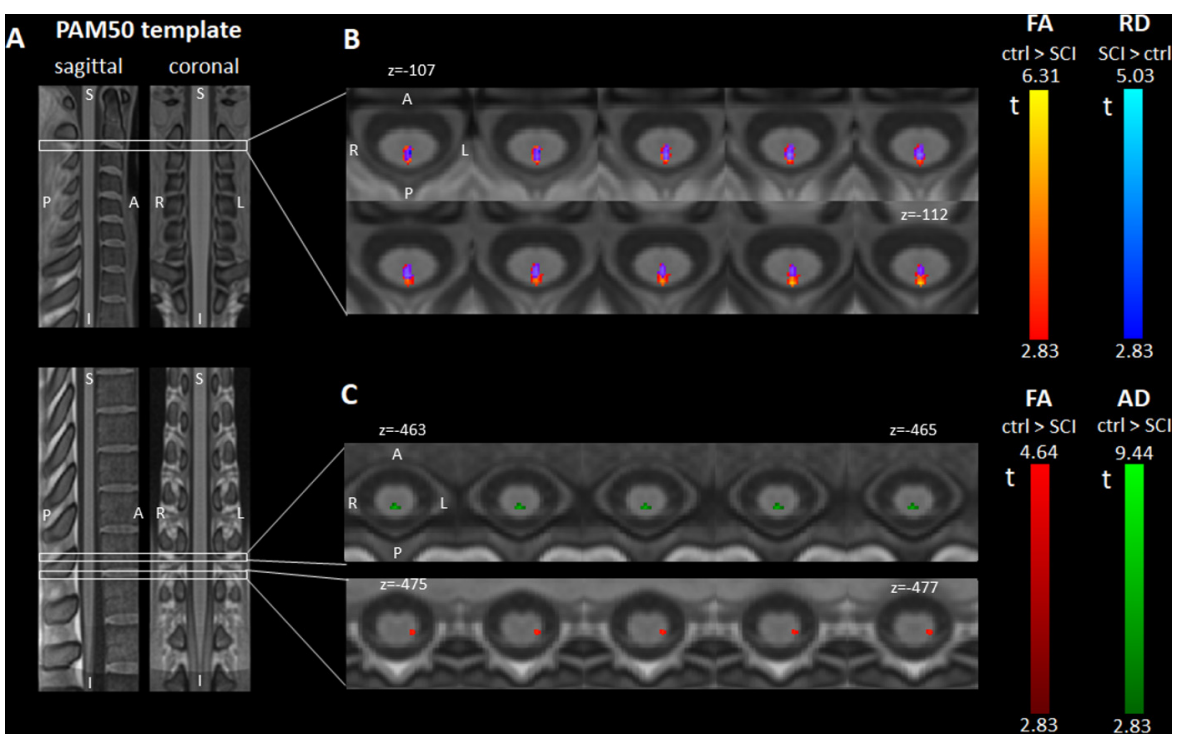

Figure 2 Differences in diffusion tensor imaging (DTI) metrics at 2 months post injury. (A) Sagittal and coronal views of the T1-weighted PAM50 spinal cord template used for the voxel-based and atlas-based DTI analyses. $(B, C)$ Axial slices of parametric maps representing the $t$ values of the voxel-based two-sample t-test comparing fractional anisotropy (FA) (displayed in red), radial diffusivity (RD) (blue) and axial diffusivity (AD) (green) between controls and patients with spinal cord injury (SCI). All parametric maps were created using SPM and are overlaid on the PAM50 template for display. The displayed clusters represent voxels with significant t values at an initial voxel-level threshold of 0.005 (uncorrected) followed by peak-level threshold of 0.05 (familywise error corrected). (B) At C2/C3 (above the lesion), significant and overlapping clusters of FA decrease and RD increase were found in the dorsal column of patients with $\mathrm{SCl}$. The overlapping region is displayed in violet. (C) In the lumbar enlargement (below the lesion), patients with $\mathrm{SCl}$ had lower $\mathrm{FA}$ in the left lateral column (corresponding to the left corticospinal tract) and lower AD in the dorsal column.

$( \pm \mathrm{SD})$ mid-sagittal lesion width, length and area were $4.9 \pm 2.2 \mathrm{~mm}$, $19.9 \pm 17.0 \mathrm{~mm}$ and $70.2 \pm 70.9 \mathrm{~mm}^{2}$, respectively, at 2 months post injury. The mid-sagittal lesion width decreased over time at a rate of $0.93 \mathrm{~mm}$ per month $(95 \% \mathrm{CI}$ : -0.16 to $-0.02, \mathrm{p}=0.016)$, while changes in lesion area and length were not significant.
Longitudinal changes in cross-sectional tissue areas

At 2 months post injury, patients had smaller SCA (controls vs SCI: 85.4 vs $\left.78.6 \mathrm{~mm}^{2},-7.9 \%, \mathrm{p}=0.008\right)$ and WMA (72.8 vs $\left.65.7 \mathrm{~mm}^{2},-9.7 \%, \mathrm{p}=0.004\right)$ at $\mathrm{C} 2 / \mathrm{C} 3$. In the lumbar enlargement, patients had smaller SCA $\left(65.0\right.$ vs $56.1 \mathrm{~mm}^{2},-13.7 \%$,

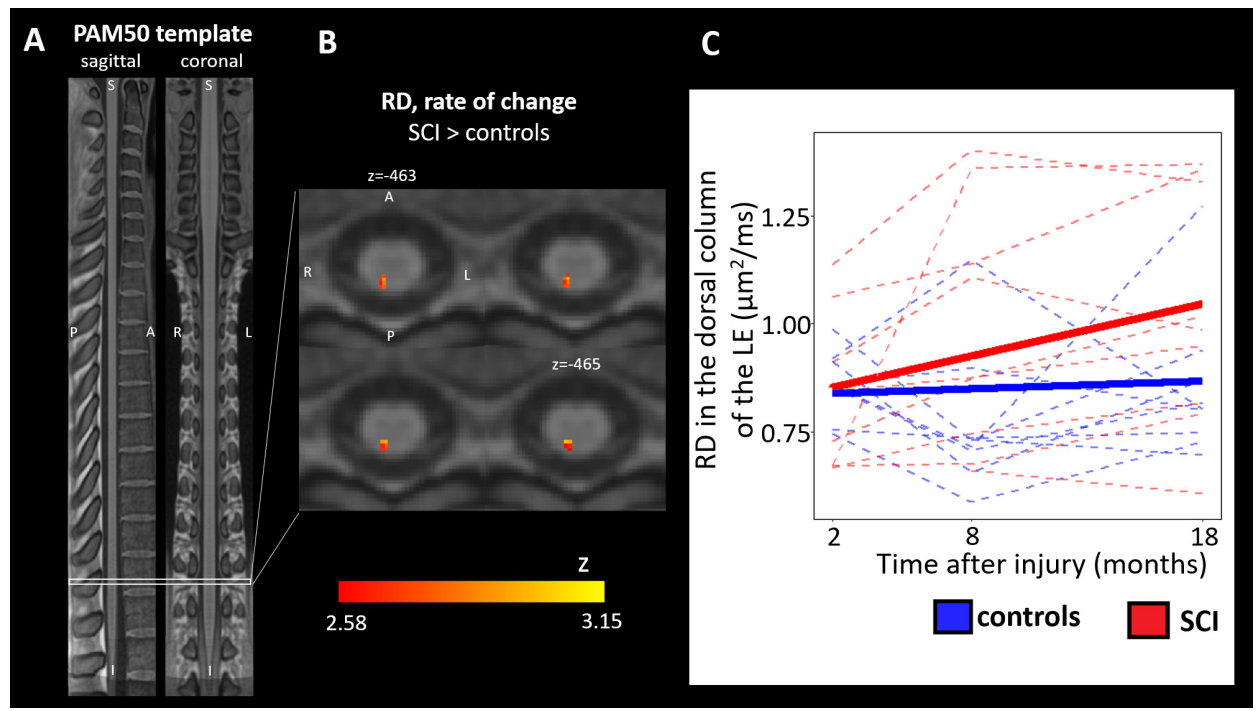

Figure 3 Longitudinal changes of diffusion tensor imaging (DTI) metrics. (A) Sagittal and coronal views of the T1-weighted PAM50 spinal cord template used for the voxel-based and atlas-based DTI analyses. (B) Axial slices of the parametric z map representing the z scores associated with the linear component of the voxel-based linear mixed effect model that models the evolution of radial diffusivity (RD) as a quadratic function over time. The displayed cluster represents voxels with significant t values at an initial voxel-level threshold of 0.005 (uncorrected) followed by peak-level threshold of 0.05 (false discovery rate corrected). In the dorsal column of patients with spinal cord injury (SCI), a significant cluster of voxels with RD increase was found in the lumbar enlargement. (C) Longitudinal changes in RD averaged within the dorsal column in the lumbar enlargement (below the injury). Dashed lines represent evolution in individual subjects, while the solid lines represent the quadratic fit in controls and patients with $\mathrm{SCl}$ separately. Patients with $\mathrm{SCl}$ showed a significant RD increase over 1.5 years after injury compared with controls. 
$\mathrm{p}=0.003)$, WMA (45.4 vs $\left.41.6 \mathrm{~mm}^{2},-8.5 \%, \mathrm{p}=0.049\right)$ and GMA (19.6 vs $\left.16.5 \mathrm{~mm}^{2},-15.9 \%, \mathrm{p}=0.001\right)$.

In controls, none of the cross-sectional area metrics changed significantly over time. Compared with controls, patients had a significantly greater rate of change (negative direction, atrophy) for a number of metrics. At C2/C3, SCA decreased at a rate of $0.42 \%$ per month $\left(0.33 \mathrm{~mm}^{2}\right.$ per month, $95 \% \mathrm{CI}:-0.47$ to -0.20 , controls vs SCI: $\mathrm{p}=0.002)$, GMA at $0.70 \%$ per month (0.09 $\mathrm{mm}^{2}$ per month, CI: -0.14 to $\left.-0.04, \mathrm{p}=0.004\right)$, and WMA at $0.34 \%$ per month $\left(0.23 \mathrm{~mm}^{2}\right.$ per month, CI: -0.35 to $-0.11, \mathrm{p}=0.020$ ) (figure $1 \mathrm{C}-\mathrm{E}$ ). In the lumbar enlargement, SCA decreased at a rate of $0.35 \%$ per month $\left(0.21 \mathrm{~mm}^{2}\right.$ per month, CI: -0.31 to $-0.10, \mathrm{p}<0.001)$ and WMA at $0.35 \%$ per month $\left(0.15 \mathrm{~mm}^{2}\right.$ per month $\mathrm{CI}$ : -0.25 to $\left.-0.05, \mathrm{p}=0.007\right)$ (figure $1 \mathrm{~F}-\mathrm{H})$.

\section{Longitudinal changes in DTI metrics}

In patients, atlas-based analysis of DTI metrics revealed lower FA (controls vs SCI: 0.68 vs $0.60,-11.3 \%, \mathrm{p}=0.008$ ) and a trend to higher RD $\left(0.63\right.$ vs $\left.0.76 \mu \mathrm{m}^{2} / \mathrm{ms},+20.5 \%, \mathrm{p}=0.059\right)$ in the dorsal column at $\mathrm{C} 2 / \mathrm{C} 3$. In the lumbar enlargement, patients had lower FA in the lateral $(0.54$ vs $0.48,-10.3 \%, \mathrm{p}=0.015)$ and ventral columns $(0.48$ vs $0.44,-9.7 \%, \mathrm{p}=0.043)$ and lower $\mathrm{AD}$ in the dorsal column $\left(2.11\right.$ vs $\left.1.83 \mu \mathrm{m}^{2} / \mathrm{ms},-13.4 \%, \mathrm{p}=0.005\right)$. Voxel-based analysis corroborated atlas-based findings: at C2/ C3, dorsal column exhibited spatially specific FA decrease (PAM50 coordinates: $x=0, y=-49, z=-106, z$ score $=4.67$, FWE-corrected $\mathrm{p}=0.002)$ and $\mathrm{RD}$ increase $(\mathrm{x}=0, \mathrm{y}=-48$, $\mathrm{z}=-109, \mathrm{z}$ score $=4.03$, FWE-corrected $\mathrm{p}=0.019$ ) (figure $2 \mathrm{~B}$ ). In the lumbar enlargement, the left lateral column had lower FA $(x=-4, y=-47, z=-476, z$ score $=3.75$, FWE-corrected $\mathrm{p}=0.021)$ and the dorsal column had lower AD $(\mathrm{x}=0, \mathrm{y}=-47$, $\mathrm{z}=-462$, $\mathrm{z}$ score $=3.75$, FWE-corrected $\mathrm{p}=0.014$ ) (figure 2C).

Controls showed no significant changes over time in any of the three ROIs in the atlas-based, nor in the voxel-based analysis. In the dorsal column of the lumbar enlargement, patients showed a trend to increasing values for RD, at a rate of $1.2 \times 10^{-2} \mu \mathrm{m}^{2} / \mathrm{ms}$ per month $\left(95 \% \mathrm{CI}: 0.3 \times 10^{-3}\right.$ to $2.2 \times 10^{-2} \mu \mathrm{m}^{2} / \mathrm{ms}$, controls vs SCI: $\mathrm{p}=0.097$ ) (figure $3 \mathrm{C}$ ), which was also confirmed in a voxel-based analysis $(x=1, y=-47, z=-474, z$ score $=3.15$, FDR-corrected $\mathrm{p}=0.083$ ) (figure $3 \mathrm{~B}$ ).

Comparison between dynamic white and grey matter atrophy At C2/C3, patients with SCI had higher standardised GMA than standardised WMA by a score of $0.86(\mathrm{p}<0.001)$ at 2 months post-injury, but this difference declined over time at a rate of 0.064 per month $(\mathrm{p}=0.014)$, completely diminishing by 1.5 years post injury (figure 4). In the lumbar enlargement, standardised GMA and WMA values were not significantly different at any timepoint (figure 4).

\section{Relationship between baseline MRI measures and 1.5-year clinical scores}

In patients with SCI, baseline ( 2 months post injury) FA values in the dorsal column at $\mathrm{C} 2 / \mathrm{C} 3$ correlated positively with the 1.5-year SCIM score, independently of the baseline SCIM score $(\mathrm{x}=-2, \mathrm{y}=-47, \mathrm{z}=-104, \mathrm{z}$ score $=4.18$, FWE-corrected $\mathrm{p}=0.037)$. In overlapping regions, baseline RD correlated negatively with the 1.5 -year SCIM score $(x=-1, y=-47, z=-103$, $\mathrm{z}$ score $=4.01$, FWE-corrected $\mathrm{p}=0.035$ ) (figure 5B). At $\mathrm{C} 2 / \mathrm{C} 3$, baseline $\mathrm{AD}$ values in the dorsal column correlated positively with the 1.5-year ISNCSCI light touch scores $(x=-1, y=-49$,

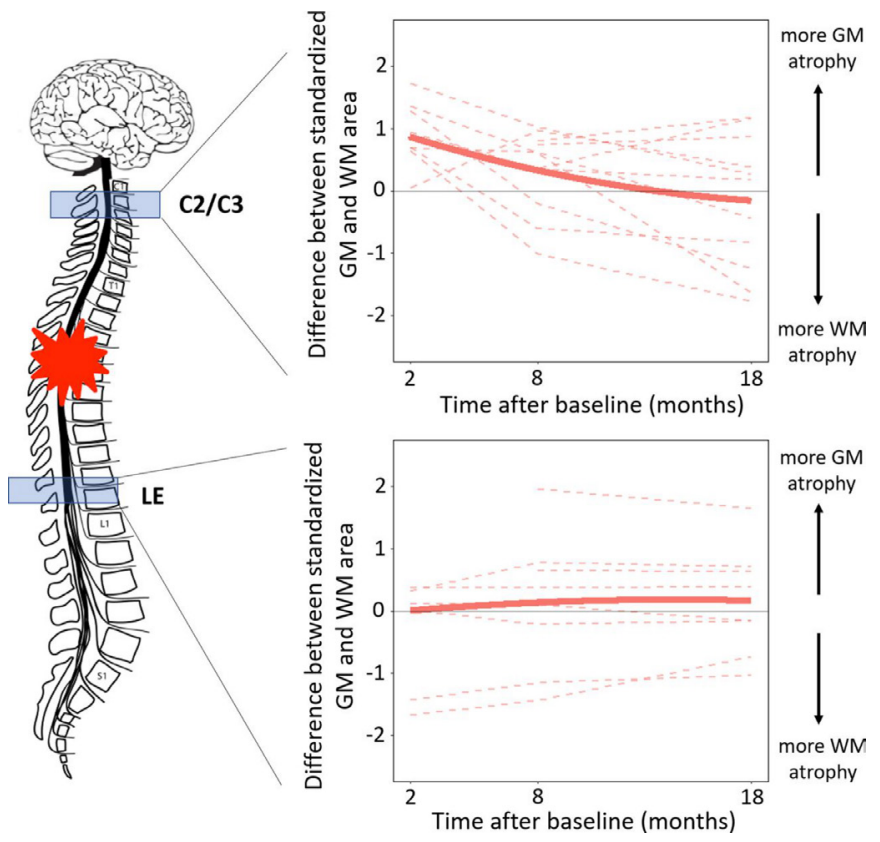

Figure 4 Longitudinal changes in the difference between spinal cord injury $(\mathrm{SCl})$ patients' standardised white matter (WM) and grey matter (GM) areas over time at C2/C3 and in the lumbar enlargement (LE).

For each patient with $\mathrm{SCl}$, the standardised WM area is calculated by subtracting the average WM area in controls and dividing the result by the SD of WM area across controls (same approach for GM area). Dashed lines represent evolution in individual subjects, while the solid line represents the quadratic fit.

$\mathrm{z}=-126$, $\mathrm{z}$ score $=4.15, \mathrm{p}=0.026$ ) (figure $5 \mathrm{C}$ ). Baseline crosssectional tissue areas did not correlate with 1.5 -year outcome scores.

\section{DISCUSSION}

This study illustrates spatiotemporal trajectories of tissuespecific spinal cord neurodegeneration, both rostral and caudal to the level of injury. Importantly, it reveals differences in the evolution of tissue-specific changes below and above the level of injury. Baseline white matter diffusion metrics predicted clinical recovery.

\section{Early changes after $\mathrm{SCI}$}

As early as 2 months after injury, white matter atrophy was detectable both rostral and caudal to the injury. Although the pattern of anterograde and retrograde degeneration is different rostral and caudal to the injury, the magnitude of white matter atrophy was very similar at both levels. Rostral to injury (at C2/ C3), the dorsal columns in patients exhibited lower FA driven by higher RD values, which has been associated with demyelination, ${ }^{15} 3132$ but without similar findings in motor tracts. This finding suggests that rostral to injury anterograde degeneration of the sensory tracts is more prominent than retrograde degeneration of the motor tracts early after injury. Caudal to injury (in the lumbar enlargement), FA was lower in the lateral and ventral tracts, while $\mathrm{AD}$ was lower in the dorsal columns, providing evidence for both anterograde degeneration of motor tracts and retrograde degeneration of sensory tracts. Interestingly, the results suggest that white matter atrophy, both at $\mathrm{C} 2 / \mathrm{C} 3$ and in the lumbar enlargement, occurs at the earliest timepoints after injury. 


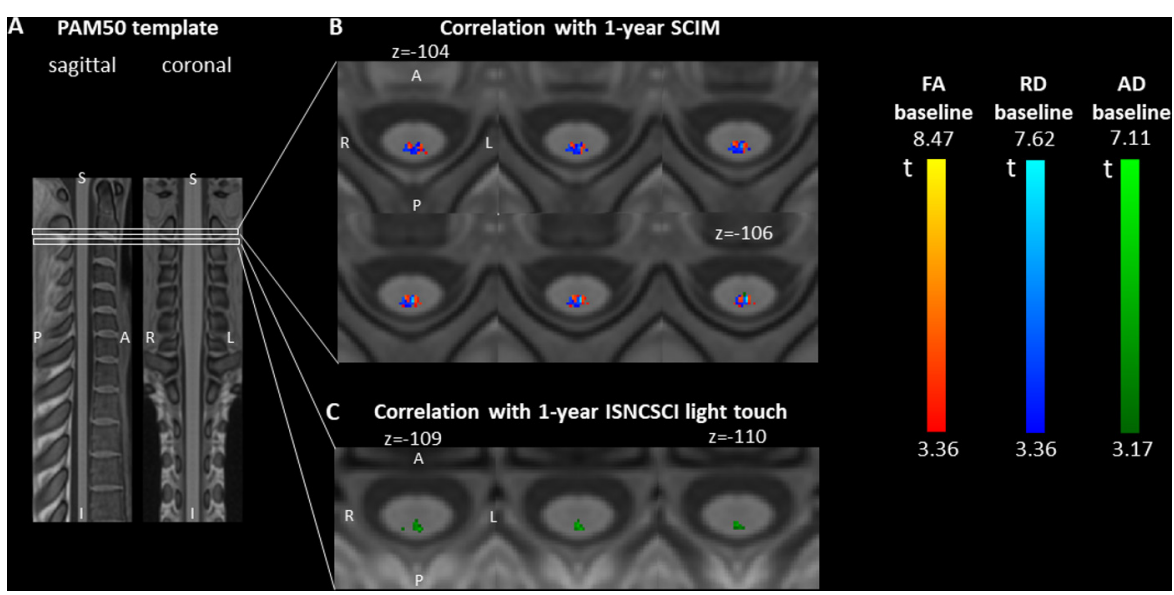

Figure 5 Associations between baseline diffusion tensor imaging (DTI) metrics and 1.5-year outcome. (A) Sagittal and coronal views of the T1-weighted PAM50 spinal cord template used for the voxel-based and atlas-based DTI analyses. (B,C) Axial slices of parametric t-maps representing the $t$ values associated with the baseline DTI values in the prediction model. The displayed cluster represents voxels with significant $t$ values at an initial voxel-level threshold of 0.005 (uncorrected) followed by a cluster-level extent threshold of 0.05 (false discovery rate corrected). The parametric map is overlaid on the T1-weighted PAM50 template. (B) In overlapping regions in the dorsal column at C2/C3, baseline fractional anisotropy (FA) and radial diffusivity (RD) in patients with spinal cord injury correlate with 1.5-year Spinal Cord Independence Measure (SCIM) score (positive correlation with FA, negative with RD), independent of baseline SCIM. (C) In the dorsal column at C2/C3, baseline axial diffusivity (AD) correlates positively with 1.5-year International Standards for Neurological Classification of Spinal Cord Injury (ISNCSCI) light touch score, independent of baseline light touch score.

\section{Longitudinal changes after $\mathrm{SCl}$}

As in previous reports investigating spinal cord neurodegeneration, ${ }^{8} 1018$ we observed a progressive remote white matter atrophy into the chronic stages of injury. Within 1.5 years after injury, white matter showed a consistent decline in tissue area, at a similar rate both at $\mathrm{C} 2 / \mathrm{C} 3$ and in the lumbar enlargement $(0.34 \%$ vs $0.35 \%)$, without significant signs of deceleration at 1.5 years post injury. At C2/C3, the observed spinal cord atrophy rate (grey and white matter combined) of $0.33 \mathrm{~mm}^{2}$ per month was in line with the previously reported value of $0.41 \mathrm{~mm}^{2}$ per month in SCI. ${ }^{8}$ In the lumbar enlargement, we did not find comparable values in the literature. Interestingly, the magnitude of white matter atrophy observed at 1.5 year post injury reached values seen in separate cohorts of chronic patients with SCI at C2/C3 $(-14.9 \% \text { vs }-16.9 \%)^{16}$ and in the lumbar enlargement $(-12.3 \%$ vs $-10.8 \%),{ }^{17}$ indicating decreasing atrophy rates after 1.5 years. Beside the progressive atrophy of white matter, the increase of RD in the lumbar enlargement over 1.5 years further supports the hypothesis that progressive neurodegeneration occurs over an extended time after injury, well into the chronic stage of injury.

While atrophy of the grey matter was only evident in the lumbar enlargement in the early stages of injury, GMA decreased within 1.5 years after injury, both rostral and caudal to the injury site. However, in contrast to white matter, we observed a discrepancy between findings at $\mathrm{C} 2 / \mathrm{C} 3$ and in the lumbar enlargement. At $\mathrm{C} 2 / \mathrm{C} 3$, grey matter showed no sign of atrophy 2 months post injury, but there was a steep decline afterwards, with GMA being already $14.4 \%$ smaller than aged-matched controls at 1.5 years post injury. In the lumbar enlargement, grey matter was considerably atrophied 2 months after injury, and exhibited only a moderate rate of progressive atrophy afterwards, which largely decelerated by 1.5 years post injury. The magnitude of grey matter atrophy in the lumbar enlargement reached at 1.5 years was similar to the value previously reported in chronic patients $(-9.6 \%$ vs $-13.0 \%),{ }^{17}$ while it was still considerably lower at $\mathrm{C} 2 / \mathrm{C} 3(-11.4 \%$ vs $-30.0 \%),{ }^{16}$ indicating continued grey matter atrophy beyond 1.5 years.

\section{Differential degenerative trajectories in grey and white matter}

The discrepancy between C2/C3 and lumbar enlargement with respect to the onset of grey matter degeneration can be explained by the differential mechanisms involved in above-level and below-level grey matter degeneration. Grey matter atrophy above the lesion is thought to occur via synapses with interneurons in a process associated with the perturbation of propriospinal network, ${ }^{33}$ which is presumably a much slower process than the transsynaptic degeneration occurring in the grey matter below the lesion. Note that other mechanisms including changes in the amount and size of glial cells and vascular changes could also be involved in grey matter atrophy; however, their degree of contribution has not yet been established. ${ }^{34}$

\section{Prognostic value of early MRI findings}

We showed that baseline MRI findings are associated with clinical recovery. Interestingly, we found that baseline DTI values are better predictors of clinical outcome than cross-sectional tissue areas, which can be explained by the large intrinsic variability and the unspecificity of tissue areas to microstructural changes. In particular, the degree of myelination in the dorsal column at $\mathrm{C} 2 / \mathrm{C} 3$ (as revealed by $\mathrm{RD}$ ) proved to be the best predictor for functional independence (ie, SCIM score), a key outcome score.

\section{Limitations}

The study included a heterogeneous patient cohort in terms of injury type, severity and lesion level. Of the three patients with non-traumatic SCI, two suffered from an ischaemic injury and one from a haemorrhage due to a cavernoma. While functional recovery trajectories after traumatic and ischaemic injuries are rather similar, ${ }^{35}$ the impact of haemorrhage on degenerative changes and recovery profiles is understudied. The heterogeneity certainly impacts the generalisability of the results; for example, we expect to see more degeneration in the lumbar enlargement at the subacute stage in patients with a lower level of injury, and more atrophy in general in a pure AIS A 
cohort. While the sample size was rather small, we primarily focused on longitudinal analyses and the number of datapoints (68) provided sufficient power for the longitudinal statistics. A potential source of bias is the manual segmentation of grey matter and semiautomatic segmentation of the spinal cord. At present, automatic segmentation algorithms have been validated only for the cervical grey matter, ${ }^{36}$ while manual segmentation continues to remain the gold standard technique in the lumbar cord. To make manual segmentation as accurate and unbiased as possible, all segmentations were performed by the same experienced user (GD) in a blinded way. Therefore, we argue that the potential segmentation bias is systematic and does not affect the longitudinal results. Another limitation is the test-retest variability of the MRI readouts, which especially impacts the inherently noisier DTI metrics (figure 3). However, since the noise component is stochastic over data points, healthy controls did not show any longitudinal changes in any metric over time. This makes us confident that the statistically significant longitudinal changes in patients represent biologically meaningful degenerative processes.

\section{Conclusion}

This study reveals distinct temporospatial dynamic trajectories of tissue-specific neurodegeneration above and below an SCI. It highlights that degeneration in the grey matter above the level of injury is initially lower than in the white matter but accelerates over time to reach similar magnitudes of atrophy by approximately 1.5 years post-SCI. Below the lesion, grey and white matter atrophy progress along similar trajectories. The clinical eloquence of these findings is reflected by the clinicopathological association between early degenerative changes and functional and neurological recovery. These MRI measures could be used to track the efficacy of therapeutic interventions, including rehabilitation.

\begin{abstract}
Acknowledgements We would like to thank Daniel Altmann for his help with the statistical analysis of time lag differences and critical revision of manuscript for intellectual content. We would also like to thank all the patients and healthy volunteers who participated in this study. We further thank the staff of the Department of Radiology and Neurology at the University Hospital Balgrist.
\end{abstract}

Contributors GD: study concept and design, data acquisition, analysis and interpretation of data, statistical analysis and writing the manuscript. DP: analysis of data and critical revision of manuscript for intellectual content. KV: data acquisition, analysis of data, interpretation of data and critical revision of manuscript for intellectual content. NP: data acquisition, analysis of data and critical revision of manuscript for intellectual content. AT and AC: study concept and design and critical revision of manuscript for intellectual content. NW and SM: study concept and design, MRI protocol implementation and critical revision of manuscript for intellectual content. PF: study concept and design, interpretation of data, writing the manuscript and study supervision.

Funding This research was supported by Wings for Life, Austria (WFL-CH-007/14), the International Foundation for Research in Paraplegia (IRP-P158 and IRP-P184), the EU project (Horizon2020 'NISCI' grant agreement no. 681094) and the framework of ERA-NET NEURON (BMBF, 01EW1711A and B). PF is funded by an SNF Eccellenza Professorial Fellowship grant (PCEFP3_181362/1). NW was supported by the European Research Council under the European Union's Seventh Framework Programme (FP7/2007-2013)/ERC grant agreement nº 616905. SM was supported by the Marie Sklodowska-Curie Individual Fellowship MSCA-IF-2015 (EU Horizon 2020) and the Deutsche Forschungsgemeinschaft (grant Number: MO 2397/4-1). Open access of this publication is supported by the Wellcome Trust (091593/Z/10/Z).

Competing interests The Wellcome Trust Centre for Neuroimaging and Max Planck Institute for Human Cognitive and Brain Sciences have an institutional research agreement with and receives support from Siemens Healthcare. NW was a speaker at an event organized by Siemens Healthcare and was reimbursed for the travel expenses.

Patient consent for publication Not required
Ethics approval The study protocol was designed in accordance with the Declaration of Helsinki and was approved by the local ethics committee (EK2010-0271). All participants provided written informed consent prior to study enrolment.

Provenance and peer review Not commissioned; externally peer reviewed.

Data availability statement Data are available upon reasonable request. Anonymised grouped data will be shared by request from a qualified investigator.

Open access This is an open access article distributed in accordance with the Creative Commons Attribution 4.0 Unported (CC BY 4.0) license, which permits others to copy, redistribute, remix, transform and build upon this work for any purpose, provided the original work is properly cited, a link to the licence is given, and indication of whether changes were made. See: https://creativecommons.org/ licenses/by/4.0/.

\section{ORCID iDs}

Gergely David http://orcid.org/0000-0002-9379-5193

Dario Pfyffer http://orcid.org/0000-0002-2406-9251

Patrick Freund http://orcid.org/0000-0002-4851-2246

\section{REFERENCES}

1 Ahuja CS, Wilson JR, Nori S, et al. Traumatic spinal cord injury. Nat Rev Dis Prim 2017;3:17018.

2 Dobkin B, Apple D, Barbeau H, et al. Weight-supported treadmill vs over-ground training for walking after acute incomplete SCI. Neurology 2006;66:484-93.

3 Vallotton $\mathrm{K}$, Huber E, Sutter $\mathrm{R}$, et al. Width and neurophysiologic properties of tissue bridges predict recovery after cervical injury. Neurology 2019;92:e2793-802.

4 Huber E, Lachappelle P, Sutter R, et al. Are midsagittal tissue bridges predictive of outcome after cervical spinal cord injury? Ann Neurol 2017:81:740-8.

5 Pfyffer D, Huber E, Sutter R, et al. Tissue bridges predict recovery after traumatic and ischemic thoracic spinal cord injury. Neurology 2019;93:e1550-60.

6 Alizadeh A, Dyck SM, Karimi-Abdolrezaee S. Traumatic spinal cord injury: an overview of pathophysiology, models and acute injury mechanisms. Front Neurol 2019;10:1-25

7 Kakulas BA. The applied neuropathology of human spinal cord injury. Spinal Cord 1999;37:79-88.

8 Freund P, Weiskopf N, Ashburner J, et al. Mri investigation of the sensorimotor cortex and the corticospinal tract after acute spinal cord injury: a prospective longitudinal study. Lancet Neurol 2013;12:873-81.

9 Jure I, Labombarda F. Spinal cord injury drives chronic brain changes. Neural Regen Res 2017;12:1044-7.

10 Grabher P, Callaghan MF, Ashburner J, et al. Tracking sensory system atrophy and outcome prediction in spinal cord injury. Ann Neurol 2015;78:751-61.

11 Seif M, Ziegler G, Freund P. Progressive ventricles enlargement and cerebrospinal fluid volume increases as a marker of neurodegeneration in patients with spinal cord injury: a longitudinal magnetic resonance imaging study. J Neurotrauma 2018;35:2941-6.

12 Onifer SM, Smith GM, Fouad K. Plasticity after spinal cord injury: relevance to recovery and approaches to facilitate it. Neurotherapeutics 2011;8:283-93.

13 Raineteau 0, Schwab ME. Plasticity of motor systems after incomplete spinal cord injury. Nat Rev Neurosci 2001;2:263-73.

14 Koshinaga M, Whittemore SR. The temporal and spatial activation of microglia in fiber tracts undergoing anterograde and retrograde degeneration following spinal cord lesion. J Neurotrauma 1995;12:209-22.

15 Buss A, Brook GA, Kakulas B, et al. Gradual loss of myelin and formation of an astrocytic scar during Wallerian degeneration in the human spinal cord. Brain 2004:127:34-44.

16 Huber E, David G, Thompson AJ, et al. Dorsal and ventral horn atrophy is associated with clinical outcome after spinal cord injury. Neurology 2018;90:e1510-22.

17 David G, Seif M, Huber E, et al. In vivo evidence of remote neural degeneration in the lumbar enlargement after cervical injury. Neurology 2019;92:e1367-77.

18 Ziegler G, Grabher P, Thompson A, et al. Progressive neurodegeneration following spinal cord injury. Neurology 2018:90:e1257-66.

19 Yiannakas MC, Kakar P, Hoy LR, et al. The use of the lumbosacral enlargement as an intrinsic imaging biomarker: feasibility of grey matter and white matter cross-sectional area measurements using MRI at 3T. PLoS One 2014;9:e105544.

20 Ashburner J, Ridgway GR. Symmetric diffeomorphic modeling of longitudinal structural MRI. Front Neurosci 2012;6:197.

21 Horsfield MA, Sala S, Neema M, et al. Rapid semi-automatic segmentation of the spinal cord from magnetic resonance images: application in multiple sclerosis. Neuroimage 2010;50:446-55.

22 Mohammadi S, Freund P, Feiweier T, et al. The impact of post-processing on spinal cord diffusion tensor imaging. Neuroimage 2013;70:377-85.

23 Mohammadi S, Möller HE, Kugel H, et al. Correcting eddy current and motion effects by affine whole-brain registrations: evaluation of three-dimensional distortions and comparison with slicewise correction. Magn Reson Med 2010;64:1047-56.

24 De Leener B, Kadoury S, Cohen-Adad J. Robust, accurate and fast automatic segmentation of the spinal cord. Neuroimage 2014;98:528-36. 
25 David G, Freund P, Mohammadi S. The efficiency of retrospective artifact correction methods in improving the statistical power of between-group differences in spinal cord DTI. Neuroimage 2017;158:296-307.

26 De Leener B, Lévy S, Dupont SM, et al. Sct: spinal cord toolbox, an open-source software for processing spinal cord MRI data. Neuroimage 2017;145:24-43.

27 Lévy S, Benhamou M, Naaman C, et al. White matter atlas of the human spinal cord with estimation of partial volume effect. Neuroimage 2015;119:262-71.

28 Kirshblum S, Waring W. Updates for the International standards for neurological classification of spinal cord injury. Phys Med Rehabil Clin N Am 2014;25:505-17.

29 Catz A, Itzkovich M, Steinberg F, et al. The Catz-Itzkovich SCIM: a revised version of the spinal cord independence measure. Disabil Rehabil 2001;23:263-8.

30 Guillaume B, Hua X, Thompson PM, et al. Fast and accurate modelling of longitudinal and repeated measures neuroimaging data. Neuroimage 2014:94:287-302.
31 Song S-K, Sun S-W, Ramsbottom MJ, et al. Dysmyelination revealed through MRI as increased radial (but unchanged axial) diffusion of water. Neuroimage 2002;17:1429-36.

32 Sun S-W, Liang H-F, Trinkaus K, et al. Noninvasive detection of cuprizone induced axonal damage and demyelination in the mouse corpus callosum. Magn Reson Med 2006;55:302-8.

33 Isa T, Ohki Y, Alstermark B, et al. Direct and indirect cortico-motoneuronal pathways and control of hand/arm movements. Physiology 2007:22:145-52.

34 Schwab ME, Bartholdi D. Degeneration and regeneration of axons in the lesioned spinal cord. Physiol Rev 1996;76:319-70.

35 Scivoletto G, Torre M, Mammone A, et al. Acute traumatic and ischemic spinal cord injuries have a comparable course of recovery. Neurorehabil Neural Repair 2020;34:723-32.

36 Prados F, Ashburner J, Blaiotta C, et al. Spinal cord grey matter segmentation challenge. Neuroimage 2017;152:312-29. 